\title{
CORRECTIONS
}

\section{Dermatology team}

In the section on "Holistic care for skin disease" in this feature (BMJ 2016;352:i1433, doi:10.1136/bmj.i1433), Sinead Langan should have been described as a consultant dermatologist not a clinical scientist. 